\title{
A call for Return to Rogers' Innovation Diffusion Theory
}

\author{
Fred Edward K. Bakkabulindi ${ }^{1}$
}

${ }^{1}$ Makerere University [E-mail: fekbakkabulindi@bams.mak.ac.ug]

\begin{abstract}
This paper argues that Rogers' Innovation Diffusion Theory (IDT) is the original theory for guiding research on innovation diffusion and/ or adoption, from which the more recent theories have been derived. Hence, the paper suggests a framework based on the IDT, and derives 14 hypotheses for future research, basing on a review of recent literature. The framework divides the correlates of diffusion and/ or adoption of innovations into three categories: individual adopter characteristics, perceived innovation characteristics and social system or organizational characteristics. In terms of individual adopter characteristics, it is hypothesized that interaction with change agents, training and cosmopolitanism positively relate to the adoption of innovations, while age and income are negatively and positively related to the adoption of innovations respectively. Gender is related to the adoption of innovations in a way that males are more apt. Regarding perceived innovation characteristics, the perceived relative advantage, compatibility, user friendliness and 'observability' are postulated to be positively related to the adoption of innovations. On organizational characteristics, it is postulated that each of organizational readiness for change, culture, size and leader's change management style is positively related to the adoption of innovations. Gaps in the studies reviewed are highlighted.
\end{abstract}

Keywords: Innovation Diffusion Theory; Everett Rogers; Adoption.

\section{$1 \quad$ Introduction}

Rogers (2003) conceptualizes an innovation as an idea, practice, or object perceived as new by an individual. According to Crossan and Apaydin (2010), definitions of the term "innovation" abound, with each definition emphasizing a different aspect of the term. They assert that the first definition of "innovation" by Schumpeter in the late 1920s stressed the novelty aspect. According to Schumpeter (cited in Crossan \& Apaydin, 2010, p. 1155), innovation is 
reflected in novel outputs: a new good or a new quality of a good; a new method of production; a new market; a new source of supply; or a new organizational structure. Thus an innovation of interest in a given study can be an ICT such as a smartphone (e.g. Putzer \& Park, 2010). The innovation could be an application of the Internet in a specific area such as the library (e.g. Nov \& Ye, 2009); government (e.g. Gupta, Dasgupta \& Gupta, 2008); social communication (e.g. Kelleher \& Sweetser, 2012); or learning (Lee, Yoon \& Lee, 2009).

The innovation of interest can even be a new managerial technique such as Customer Relations Management, CRM (e.g. Hung, Hung, Tsai \& Jiang, 2010) or Evidence Based Practice (Aarons, Sommerfield \& Walrath-Greene, 2009). Thus as Rogers (2003) observes

...it matters little, so far as human behaviour is concerned, whether or not an idea is 'objectively' new as measured by the lapse of time since its first use or discovery. The perceived newness of the idea for the individual determines his or her reaction to it. If an idea seems new to the individual, it is an innovation (p. 12).

Adoption, according to Rogers (2003) is preceded by the diffusion of an innovation. He goes on to define "diffusion" as a "process in which an innovation is communicated through certain channels overtime among members of a "social system"" (p. 5), where a 'social system' is a "set of interrelated units that are engaged in joint problem solving to accomplish a common goal. The members or units of a 'social system' may be individuals, informal groups, organizations and/ or subsystems" (Rogers, 2003 p. 23). In other words, a social system is an organization of interest.

Rogers (2003) asserts that "diffusion is as special type of communication in which messages are about a new idea. This newness of the idea in the message content gives diffusion its special character" (p. 6). He further stresses that "diffusion is a kind of 'social change', defined as a process by which alteration occurs in the structure and function of a social system. When new ideas are invented, diffused, and adopted or rejected, leading to certain consequences, social change occurs" (p. 6). "Adoption" according to Rogers (2003), is the "decision to make full use of an innovation as the best course of action available. Rejection is a decision not to adopt an innovation" (p. 177).

Given that the process of diffusion and/ or adoption of innovations is always slow (Rogers, 2003, p. 61), one goal of diffusion and/ or adoption research is to try to expedite the process. One way to expedite the process is to identify its correlates, that is the factors affecting it, which correlates can be manipulated to positively influence the diffusion and/ or adoption of the innovation in question (Rogers, 2003). In deriving the correlates of innovation diffusion and/ or adoption, several frameworks are available. Of these frameworks, this paper 
was intended (i) to give a full account of the Innovation Diffusion Theory (IDT); (ii) to critique the more recent technology adoption models, namely the Technology Acceptance Model (TAM), the Technology-OrganisationEnvironment (TOE) Framework and the Unified Theory of Acceptance and Use of Technology (UTAUT), and to argue that all of them are derivatives of the IDT.

Hence the third and last objective of the paper was, (iii) to make a call for a return to the IDT as the original theory for guiding research on innovation diffusion and/ or adoption by suggesting a framework based on the IDT, and to derive 14 hypotheses for future research, basing on a review of recent empirical literature. This is an answer to Crossan and Apaydin (2010) who lament that "fragmentation of the field [of innovation diffusion and/ or adoption] prevents us from seeing the relations between these facets and ultimately impedes the consolidation of the field" (p. 1154). It is in line with Everett Rogers (19312004) who always argued that diffusion, and hence adoption, was a general process, not bound by the type of innovation studied, by who the adopters were, or by place or culture (Rogers, Singhal \& Quinlan, 2009).

\section{Theoretical Review}

\subsection{Innovation Diffusion Theory}

While there are several frameworks for guiding innovation diffusion and/ or adoption studies, it is argued in this paper that most of them are derived from Rogers' Innovation Diffusion Theory (IDT), which is dealt with in this Subsection 2.1. And although referred to as Rogers Innovation Diffusion Theory (IDT) in this paper, the theory proposed by Rogers in 1958 after his doctoral studies in the diffusion of agricultural innovations (Rogers, 1957) at Iowa State University, US, is officially termed the "Paradigm of InnovationDecision Process" (Rogers, 2003). It is also variously known as the Classical Innovation Theory (Hung et al., 2010); the Diffusion of Innovations (Kelleher \& Sweetser, 2012); and the Diffusion Theory (Kelleher \& Sweetser, 2012), among others.

According to Rogers (2003), the IDT relates innovation diffusion and/ or adoption to three categories of correlates, namely the characteristics of the individual potential adopter, how the adopter perceives the innovation, and the characteristics of the social system or organization where the potential adopter is. Regarding the individual characteristics of the potential adopter as correlates of innovation diffusion and/ or adoption, Rogers stipulates that an individual's 
propensity to adopt or use any innovation such as ICT, depends on the individual characteristics of that person.

Such individual adopter characteristics include the extent to which that person interacts with the change agents of relevance to the innovation in question; the level of training of relevance to the innovation the person has received; how cosmopolitan (i.e. urban influenced or non-conservative) the person is; how old the person is; the gender and the income level of the person. If the person interacts much with the change agents of relevance to the innovation in question, then that person will have a high propensity to adopt the innovation. If the person has a high level of training of relevance to the innovation, then that person will have a high propensity to adopt the innovation. If the person is cosmopolitan, then that person will have a high propensity to use the innovation. The older a person becomes, the less that person will be attracted to adopt innovations. In terms of the gender, the males are usually more apt to use innovations than the females. The wealthier a person becomes, the more able that person will be to acquire and hence to adopt innovations.

With respect to the perceived characteristics of the innovation as correlates of innovation adoption, Rogers' IDT stipulates that an individual's propensity to adopt or use any innovation, depends on the way that individual perceives the innovation in terms of such issues as its relative advantage, compatibility, user friendliness and 'observability'. If the individual perceives the innovation to have relative advantage over similar products or services say in terms of speed of performance, then that individual will have a high propensity to adopt the innovation. If the individual perceives the innovation to be compatible with the individual's work and interests, then that individual will have a high propensity to adopt the innovation. If the individual perceives the innovation to be user friendly, then that individual will have a high propensity to adopt the innovation. If the individual perceives the innovation to be observable, that is to have observable impact on the work of colleagues, then that individual will have a high propensity to adopt the innovation.

Lastly, on the nature of the social system or organizational characteristics as correlates of innovation diffusion and/ or adoption, Rogers (2003) stipulates that an individual's propensity to adopt or use any innovation, depends on the organization where that individual is. That is whether the social system or organization is ready for change; whether the social system or organization has a good culture that facilitates change; whether the size of the social system or organization is fit for change; whether the leader of the social system or organization is for change and facilitates change. The more positive the answers to these questions, the easier it will be for an individual in that organization to adopt change. Several researchers have used the IDT as the theoretical basis for their studies, having categorically stated that they were doing so (e.g. Kelleher $\&$ Sweetser, 2012). 
Other researchers have used the IDT as the theoretical basis of their empirical studies without explicitly saying so (e.g. Norton, 2012). Others (e.g. Buabeng-Andoh, 2012) have used the IDT as the basis for their theoretical/ conceptual papers. Several researchers have used the IDT in combination with other theories as the theoretical basis of their empirical studies after categorically saying that they were doing so (e.g. Hung et al., 2010 combined it with the Technology-Organisation-Environment, TOE - detailed in Section 2.3). Clearly then, the IDT is very popular except that it has several names and some researchers who suggest that the TAM is far more popular than the IDT, seem to discount the fact that the IDT is used under several aliases.

\subsection{Technology Acceptance Model}

Apart from Rogers' Innovation Diffusion Theory (IDT), many other theories have been advanced to serve as frameworks for innovation diffusion and/ or adoption studies. Such theories include the all popular Technology Acceptance Model (TAM). The TAM was developed by Davis (1989) after his doctoral studies at the Sloan School of Management, Massachusetts Institute of Technology (Davis, 1986) to provide an explanation of the determinants of user acceptance of technology such as computers and the Internet. Herein lies the first major weakness of the TAM in comparison to the IDT, in that the TAM purports to deal with technological innovations only, yet not all innovations are technological. Crossan and Apaydin (2010) put it very well when they observe that innovations take three basic types, namely product, process or business model. In the TAM, Davis proposed that the perceived ease of use (PEU) and the perceived usefulness (PU) of a technology, are the two factors that affect an individual's behavioural intention (BI) to use the technology, which in turn affects the actual use.

Davis (1989) defined the PEU of a technology as the degree to which a prospective user expects the technology to be free of effort. He also defined the PU of a technology as the prospective user's subjective probability that using the technology will increase the user's job performance. In short, the TAM postulates that the PU and PEU affect the BI to use a technology, which in turn affects the actual use. However as explained in Sections 5.1 and 5.3 of this paper, the reality is that in the TAM, Davis simply postulates what Rogers stipulated, to the effect that the perceived relative advantage (PRA) and the perceived user friendliness (PUF) under different names namely, the perceived usefulness (PU) and the perceived ease of use (PEU) affect the behavioural intention (BI) to use a technology, which in turn affects the actual use.

Thus Davis simply extracted a very parsimonious theory from Rogers IDT. Then, who takes the primary credit for the TAM? The one extracting (Davis, $1986 ; 1989)$ or the one (Rogers' IDT) from whom the TAM was extracted? 
Despite being a derivative of the IDT, the TAM is so popular that many papers on technology adoption, both theoretical and empirical will not forget to heap praises on it. For example, Putzer and Park (2010) observe that the TAM is probably the most popular theory explaining user acceptance and behaviour related to new technologies. Thus there is a large number of studies that have used the TAM as a theoretical basis explaining the diffusion and/ or adoption of different innovations (e.g. Lule, Omwansa \& Waema, 2012). Others (e.g. Awa et al., 2012) have carried out literature reviews of empirical studies that used the TAM as their theoretical basis.

But why is the TAM so popular? According to Korpelainen (2011), the advantage of focusing on TAM is that it is a simple and parsimonious model, which has encouraged researchers to apply it widely. Ironically the TAM's major strength of parsimony, is its major shortcoming at the same time as per critics. For example, Awa, Ukoha and Emecheta (2012) contend that "although TAM has received empirical validation, applications, and replication..., the model provides less meaningful information on users' opinions about adopting specific systems by narrowing its constructs to only [perceived usefulness] PU and [perceived ease of use] PEOU...." (p. 573). Awa et al. (2012) go on to summarise their critique of the TAM thus, "TAM is accused of... technological determinism, and techno-centric predictions...." (p. 575). But as a sign of fear to fully critique the all-powerful TAM, instead of calling for a return to the IDT that the TAM abstracted, they called for expansion of the TAM, thus, "hence the need to expand the factors [in TAM] or integrate [it] with other IT acceptance models to improve TAM's explanatory and predictive utilities" (pp. 573-574).

They further called for the use of "strengths of Rogers' ... Diffusion of Innovations [Theory of Rogers]... to enrich TAM by... placing premiums on specific settings and external variables that influence a technology's adoption process" (p. 574). But surely, does enriching the TAM not simply mean a return to the IDT? Other critiques of the TAM include Gupta et al. (2008) who observe that by "only focusing on PU and PEOU... [TAM] may not be enough" (p. 145) to explain innovation diffusion and/ or adoption. Hence despite its popularity, most studies have not used the TAM in isolation. On the contrary, they have used modifications of the TAM or the TAM in conjunction with other theories as the theoretical basis explaining the diffusion and/ or adoption of different innovations (e.g. Putzer \& Park, 2010 triangulated it with the IDT).

Others (e.g. Awa et al., 2012) have reviewed empirical studies that used both the TAM and the TOE as their basis. In summary, it may suffice to observe that many researchers have suggested that the TAM needs to be given additional variables to provide an even stronger model. But to be more truthful, instead of calling for extension of the TAM, the thesis in this paper is that, they should be 
calling for a return to Rogers' Innovation Diffusion Theory (IDT) which goes beyond the TAM's perceived usefulness (PU) and the perceived ease of use (PEU), by adding other perceived innovation characteristics such as the perceived compatibility (PC) and the perceived 'observabilty' (PO) of an innovation. Rogers' IDT also adds individual characteristics and organizational characteristics as correlates of innovation diffusion and/ or adoption.

\subsection{Technology-Organisation-Environment Framework}

The Technology-Organisation-Environment (TOE) Framework is one of the many other theories than the Innovation Diffusion Theory (IDT) and the Technology Acceptance Model (TAM) that have been advanced to serve as frameworks for innovation diffusion and/ or adoption studies. The TOE was developed by Tornatzky and Fleischer (1990) to provide an explanation of the determinants of user acceptance of technology such as computers and the Internet. Like the TAM, the TOE has a shortcoming in comparison to the IDT, namely that the TOE is biased toward the technological innovations only, yet not all innovations are technological (Rogers, 2003). The TOE relates innovation adoption to three categories of correlates, namely the characteristics of the technology (read innovation) being adopted, and the characteristics of the organization where the potential adopter is, plus the characteristics of the environment, where the potential adopter's organization is.

Regarding the characteristics of the technology or innovation being adopted, the "technological context" of the TOE stipulates that the "adoption depends on... perceived relative advantage (gains), compatibility (both technical and organizational), complexity (learning curve), 'trialability'... and 'observability'" of the technology (read innovation) (Awa et al., 2012, p.574). Clearly then, in the "technological context", the TOE is restating what Rogers' IDT stipulates as the "perceived characteristics of the innovation" being correlates of innovation diffusion and/ or adoption. Who then takes the credit for this assertion? The one originally suggesting it (Rogers) or the ones just modifying the names of the variables in the assertion (Tonatzky \& Fleischer, 1990)? With respect to the "organizational context", the TOE stipulates that the diffusion and/ or adoption of an innovation depends on an organisation's "top management support, organizational culture, complexity of managerial structure..., and size" (Awa et al., 2012 p. 574) among other correlates.

Again here, in the "organisational context", the TOE (Tornatzky \& Fleischer, 1990) is restating what Rogers' IDT stipulates as "organisational characteristics" as correlates of innovation adoption. Again, who takes the credit for this thesis? It is argued in this paper that credit should go to the one who originally suggested it (Rogers) and not the ones who just modified the names of constructs in it (Tonatzky \& Fleischer, 1990). Regarding the 
organizational environment as a correlate of innovation diffusion and/ or adoption, the "environment[al] context" of the TOE relates to the "facilitating and inhibiting factors" in the area of operations. Significant amongst them are the competitive pressure, the trading partners' readiness for the innovation in question, socio-cultural issues, government encouragement, and technology support infrastructures such as the access to quality ICT consulting services (Awa et al., 2012).

Thus the only difference between the TOE and the IDT is that the former omits the "individual characteristics" of a potential adopter, while also separating the "environmental characteristics" from the "organizational characteristics". But in dropping the individual adopter characteristics, the TOE is thus inferior to the IDT as a framework for guiding researchers in identifying potential correlates of innovation diffusion and/ or adoption. Nevertheless, several studies have used the TOE framework as their theoretical basis, as summarized by Oliveira and Martins (2011). However the ones that the author of this paper has so far come across, have used the TOE framework in combination with other theories, some having categorically said that they were doing so. For example, Hung et al. (2010) combined it with the IDT.

\subsection{Unified Theory of Acceptance and Use of Technology}

One of the latest frameworks for innovation diffusion and/ or adoption studies is Venkatesh, Morris, Davis and Davis (2003)'s Unified Theory of Acceptance and Use of Technology (UTAUT). Like the TAM and the TOE framework, the UTAUT has a shortcoming in comparison to the IDT, namely that it (the UTAUT) is biased toward the technological innovations only, yet not all innovations are technological (Rogers, 2003). According to Williams, Rana, Dwivedi and Lal (2011), the UTAUT was developed through the review, mapping and integration of eight dominant theories and models. The theories and models considered were the Theory of Reasoned Action (TRA), the Technology Acceptance Model (TAM), the Motivational Model (MM), the Theory of Planned Behaviour (TPB), a combined Theory of Planned Behaviour/ Technology Acceptance Model (C-TPB-TAM), the Model of PC Utilisation (MPCU), the Innovation Diffusion Theory (IDT) of Rogers, and the Social Cognitive Theory (SCT).

The UTAUT relates innovation diffusion and/ or adoption to four core constructs, namely the "performance expectancy" (PE), the "effort expectancy" (EE), the "social influence" (SI) and the "facilitating conditions" (FC). The UTAUT also assumes that the effect of the core constructs is moderated by the gender, age, and experience of a potential user and the voluntariness of use of the innovation. It should be noted however, that the UTAUT's "performance expectancy" (PE) construct, as explained in Subsection 5.1 in this paper, is just 
another term for Rogers' "perceived relative advantage" (PRA) of an innovation. Similarly as explained in Subsection 5.3, the UTAUT's "effort expectancy" (EE) construct, is just another term for the opposite of Rogers' "perceived complexity", namely the "perceived user friendliness" (PUF) of an innovation. Also as explained in Subsections 4.1 and 5.2 respectively in this paper, the UTAUT's "social influence" (SI) and "facilitating conditions" (FC) constructs, are respectively synonymous with Rogers" "interaction with change agents" and "perceived compatibility" (PC) of an innovation.

In short, in postulating performance expectancy (PE), effort expectancy (EE), social influence (SI) and facilitating conditions (FC) as correlates of innovation diffusion and/ or adoption, the UTAUT is simply restating what Rogers' IDT postulates, namely that the extent to which a potential user perceives an innovation to have (perceived) relative advantage (PRA) and user friendliness (PUF); plus the fact that the potential user's extent of interaction with change agents of relevance and the extent to which the user perceives the innovation to have (perceived) compatibility (PC), are all correlates of the diffusion and/ or adoption of the innovation in question. Thus the UTAUT is a very parsimonious theory extracted from Rogers IDT.

Again, the question is that, who takes the primary credit for the UTAUT? Rogers who originally suggested the four independent variables in the UTAUT as correlates of innovation diffusion and/ or adoption, or Venkatesh et al. (2003) who just modified their names? Studies basing on the UTAUT as their theoretical basis can be found (e.g. Gupta et al., 2008). However from their analysis of 450 articles, Williams et al. (2011), reported that, "although a large number of studies have cited the [UTAUT] originating article [Venkatesh et al., 2003] since its appearance, only 43 actually utilized the theory or its constructs in their empirical research" (p. 231), implying that so far most researchers just cite the UTAUT instead of actually using it.

\section{Conceptual Framework}

The critical theoretical review in Section 2 has brought out the fact that Roger's Innovation Theory (IDT) and its many aliases such as the "Paradigm of Innovation-Decision Process", the Classical Innovation Theory, the Diffusion of Innovations, and the Diffusion Theory, among others is the original theory to guide studies on the diffusion and/ or adoption of innovations. It is also more elaborate in so far as it considers the "technological, individual, organizational and institutional factors" (Buabeng-Andoh, 2012) when examining innovation diffusion and/ or adoption, while the TAM for example, considers only the technological factors and only two of them at that. It was also revealed that the 
TAM, the TOE and the UTAUT frameworks are derivatives of the IDT. Thus instead of for example calling for the use of the "strengths of Rogers' ... Diffusion of Innovations... to enrich TAM" (Awa et al., 2012, p. 574), this paper is boldly calling for a return to the classical innovation diffusion and/ or adoption theory, namely Rogers' Innovation Diffusion Theory (IDT).

Hence Figure 1 provides a framework, a scheme of concepts (variables or constructs) derived from Rogers' IDT (Subsection 2.1) which the researchers on the diffusion and/ or adoption of innovations, can operationalize in order to achieve their objectives. The framework has one dependent variable (DV), namely the "diffusion and/ or adoption of an innovation", being related to three groups of independent variables (IVs), namely the individual adopter characteristics as the first IV (IV1); the perceived characteristics of an innovation as the second IV (IV2); and the "nature of the social system" as the third IV (IV3). The term the "nature of the social system" is a synonym from Sociology for organizational characteristics. The DV was "operationally defined", that is, broken into two "concepts", namely "knowledge" and "use" of the innovation. Why? Because Rogers (2003) observes that "newness in an innovation need not just involve new knowledge.

Someone may have known about an innovation for some time but not yet developed a favourable or unfavourable attitude toward it, nor have adopted or rejected it. 'Newness' of an innovation may be expressed in knowledge, persuasion [or attitude], or a decision to adopt [or use it]" (p. 12). Rogers (2003) thus suggested that "knowledge", "attitude" and "behaviour" are suitable measures of innovation diffusion and/ or adoption. Also, Rogers (2003, p. 69) observes that "K [for knowledge], A [for attitude] and $\mathrm{P}$ [for practice or behaviour] are the main dependent variables in the evaluation of family planning programmes". However since one of the independent variables, perceived innovation characteristics, is very near to "attitude", in the model (Figure 1) it is proposed that only "knowledge" and "use" be the appropriate measures of diffusion and/ or adoption of an innovation.

Similarly the first IV (IV1) is "operationalised" into four "concepts" or individual adopter characteristics, namely the interaction with change agents of relevance to the innovation, training with respect to the innovation, cosmopolitanism (or urban influence) and demographic variables. The demographic characteristics are in turn "operationalised" as age, gender and income level. Under IV2, there are four "concepts" or perceived characteristics of the innovation, namely its perceived relative advantage, compatibility, user friendliness and 'observability'. Under IV3, there are four "concepts" or organisational characteristics, namely the organizational readiness for change, culture with respect to the innovation, size, and leader's change management style with respect to the innovation. What follows in sections 4 through 6 is a 
systematic derivation of 14 hypotheses based on the conceptual framework (Figure 1).

Independent Variables

\begin{tabular}{|l|}
\hline Individual Adopter Characteristics (IV1) \\
Interaction with change agents of \\
relevance to the innovation \\
Training with respect to the innovation \\
Cosmopolitanism \\
Demographic variables \\
- Age \\
- Gender \\
- Income level
\end{tabular}

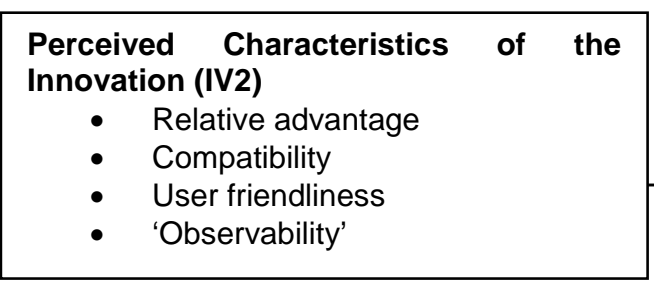

Nature of the Social System (IV3)

- Readiness for change

- Culture with respect to the innovation

- Size

- Leader's change management style with respect to the innovation

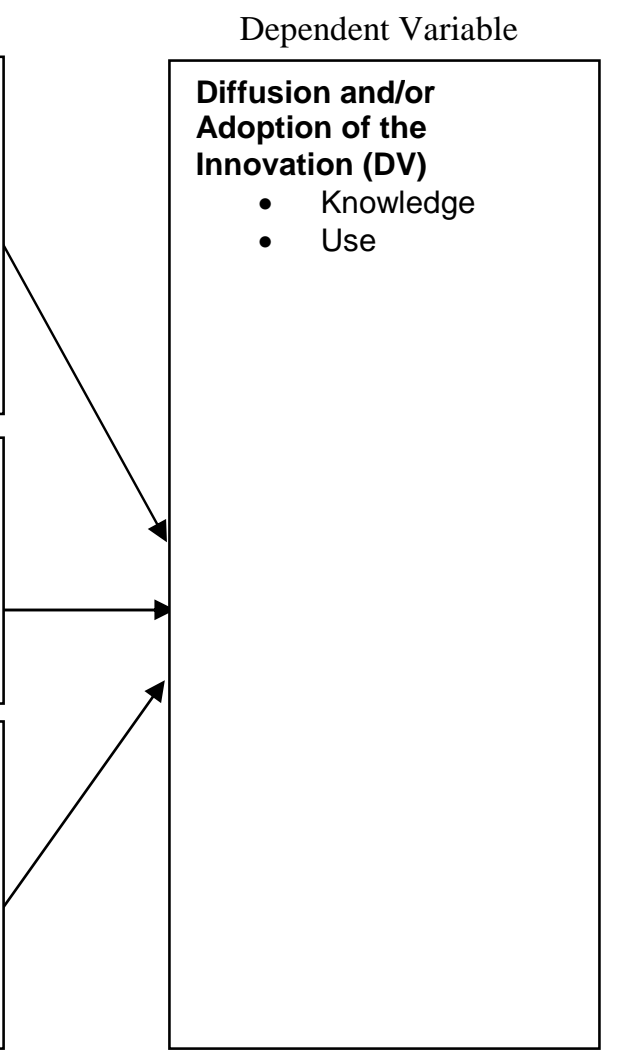

Figure 1: Conceptual model relating the adoption of innovations to three social correlates

Source: Adapted from Rogers (2003)

\section{Hypotheses on Individual Characteristics as Correlates of Adoption of Innovations}

\subsection{Interaction with Change Agents as a Correlate of Adoption of Innovations}

Rogers (2003) defines a change agent as an "individual who influences clients' innovation-decisions in a direction deemed desirable by a change agency" (p. 366). Rogers goes on to observe that many different occupations fit that definition of change agent: teachers, consultants, public health workers, agricultural extension agents, development workers, and sales people, all of whom "provide a communication link between a resource system with some 
kind of expertise and a client system" (p. 368). Rogers defines another term which seems synonymous with a "change agent", namely that of a "champion", as a "charismatic individual who throws his or her weight behind an innovation, thus overcoming indifferences or resistance that the new idea may provoke in an organisation" (p. 414). Stuart, Mills and Remus (2009) define champions as individuals who emerge to take creative ideas and bring them to life; who actively and enthusiastically promote an innovation, building support, overcoming resistance and ensuring that the innovation is implemented (p. 734).

On the importance of a change agent in the process of introducing innovations in a social system, Rogers (2003) suggests several roles of a change agent, including (i) to develop a need for change (ii) to diagnose problems (iii) to create an intent to change in the client (iv) to translate the intent into action and (v) to stabilize adoption and prevent discontinuance. However, both Rogers (2003) and Stuart et al. (2009) concur when they observe that mere presence of change agents or champions is not adequate to facilitate innovation diffusion and/ or adoption, unless certain conditions are met. On their part for example, Stuart et al. (2009, p. 734) observe that change agents or champions must "communicate a clear vision of an innovation, display enthusiasm for the innovation, demonstrate commitment and involve others in supporting it", and must exude "confidence, persistence, energy and risk-taking [which] are... key characteristics of champions".

They should "have a variety of working experiences and a long tenure in the organization to draw from... [which] helps them to understand the potential of [say] new technology while giving them a broad social network to help implement and support the change....". Recent studies (e.g. Norton, 2012) positively relating the interaction with change agents or champions and the use of innovations can be found. But so are those (e.g. Bakkabulindi \& Kabasiita, 2012) not doing so. Thus whether the "interaction with change agents or champions" is a positive correlate of the adoption of innovations is not fully clear. Hence in this paper, it is being proposed that future studies still have to test the hypothesis that interaction with change agents is a positive correlate of the adoption of innovations.

\subsection{Training as a Correlate of Adoption of Innovations}

Hong, Hao, Kumar, Ramendran and Kadiresan (2012) define "training" as the systematic acquisition and development of knowledge, skills and attitudes required by employees to adequately perform assigned tasks to boost their performance on the job. Thus, training in a work situation is concerned with extending and developing employees' capabilities and enabling them to perform better in their jobs, and be more ready for changes (Salleh, Yaakub \& 
Dzulkifli, 2011). Buabeng-Andoh (2012) on his part, observes that teachers' professional development is a key factor to their successful integration of computers into classroom teaching. He cites several studies as having revealed that whether beginner or experienced, ICT-related training programs develop teachers' competencies in computer use, influencing the teachers' attitudes towards computers as well as assisting the teachers to recognize the task of technology and how new technology tools are significant in student learning. Several recent researchers (e.g. Hung et al., 2010) have established training to be a positive correlate of the adoption of innovations. However studies not supporting the postulate (e.g. Bakkabulindi \& Kabasiita, 2012) are available. Thus the support for the assertion to the effect that "training is a positive correlate of the adoption of innovations" is not unanimous. Hence in this paper, it is being proposed that future studies still have to test the hypothesis that training is a positive correlate of the adoption of innovations.

\subsection{Cosmopolitanism as a Correlate of Adoption of Innovations}

Rogers (2003) defines "cosmopoliteness", another version of the term "cosmopolitanism" as the "degree to which an individual is oriented outside a social system" (p. 290). He observes that early adopters' or innovators' interpersonal networks are more likely to be outside, rather than within, their social system. That they travel widely and are involved in matters beyond the boundaries of their local system. The innovators act like the "stranger", whose special perspective stems from a lack of integration into the local system. The "stranger" is not radically committed to the unique ingredients and peculiar tendencies of the group, and because of this social distance from others in the social system, the "stranger" is relatively free from the system's norms. This orientation frees the innovator from the constraints of the local system and allows him or her personal freedom to try out previously untried ideas.

In this paper, it is assumed that "cosmopoliteness" is synonymous with "urban influence". Rogers (2003) asserts that the earlier adopters or innovators are more "cosmopolite" or cosmopolitan than the late adopters or noninnovators. Recent studies positively relating "cosmopoliteness" or urban influence and the diffusion and/ or adoption of innovations can be found (e.g. Billon, Macro \& Lera-Lopez, 2009). But many of these past studies relate to ICT innovations. Hence it is still incumbent on future studies especially those on other innovations than ICT to still test the research hypothesis to the effect that cosmopolitanism is a positive correlate of the adoption of innovations.

\subsection{Age as a Correlate of Adoption of Innovations}

Schiffman and Kanuk (2004) observe that the age of the consumer innovator is related to the specific product category in which the consumer innovates, with 
the consumer innovators tending to be younger than the late adopters or noninnovators because many of the products selected for research attention such as fashion and automobiles, are particularly attractive to the young consumers. Awa et al. (2012) explain the reluctance of the older executives to adopt ecommerce (EC), and by implication, other innovations, thus:

The conservative stance of the older executives is explained by their premiums on social circles and spending traits, retirement benefits, and career and financial security...; lack of mental and physical stamina to grasp novelties; greater psychological commitment to the corporate status-quo...; and the lack of the social enabling environment for novelties.... Therefore, the likelihood of EC adoption [or the adoption of any other innovation] is more profound in organisations managed by young executives than those managed by the older executives (p. 578).

Several recent studies (e.g. Bakkabulindi, 2011) have established age to be a direct negative correlate of the diffusion and/ or adoption of innovations. But some recent studies (e.g. Educause Centre for Applied Research, ECAR, 2010) did not find age to correlate with the use of innovations at all. Yet other recent studies (e.g. Billons et al.., 2009) have found age to actually be a positive correlate of the diffusion and/ or adoption of innovations. Thus as Rogers (2003) observes, "there is inconsistent evidence about the relationship of age and innovativeness" (p. 288), that is the readiness for an individual to adopt innovations. It is thus a long time in the future when researchers will know for sure whether age is inversely related to the adoption of innovations.

\subsection{Gender as a Correlate of Adoption of Innovations}

Gender refers to the socially constructed differences and distinctions between men and women. Gender differs from sex in that it is not biologically determined. Gender distinctions include the different attributes, statuses, roles, responsibilities, and potentialities as well as their access to and the control over resources and benefits (Ssali, Ahikire \& Madanda, 2007). Dlodlo (2009) summarized the attitudes expressed by many authors about technology in general and ICT in particular as being a male-dominated discipline, saying that:

the dominant cultural understanding of technology is as a masculine activity, therefore women have often chosen not to engage in it.... Traditionally, anything... difficult to perform is considered the preserve of the male species. Therefore, girl children would psychologically have a barrier taking up science subjects, including ICT.... There is a shortage of role models who have succeeded in ICT careers and can be emulated among the women folk... Boys have more access to technology at home than girls.... In this 
light, boys are encouraged and have more positive attitudes towards ICTs than girls (p. 172).

Dlodlo (2009 p. 173) also captures very well the phenomenon of how women's responsibilities for family life curtail their abilities to be as technology-savvy as their male counterparts, thus:

Women are responsible for family life. The triple workloads of domestic, income generation, and community management activities mean that women often do not have free time to travel, learn about, and use ICT. It is a challenge [for them] to balance family life and [ICT] training....

Sang, Valcke, van Braak and Tondeur (2010) while acknowledging the general belief that ICT is a male domain, do not fully concur with the observations by Dlodlo (2009), when they contend that:

[the] literature on educational computing abounds with conflicting findings about the impact of gender.... Since the introduction of computers, ICT related activities have been viewed as a 'male domain'.... There is a significant body of evidence supporting the notion that gender plays a role in actual computer integration.... [However] since technologies have become a normal part of the working place setting, a number of researchers argue that computing should no longer be regarded as a male domain (p. 104).

Recent studies (e.g. Dlodlo, 2009) revealing that the males were more apt on the adoption of innovations than women, can be found. However, there are also recent studies (e.g. Sang et al., 2010), whose findings totally dismissed gender as a correlate of innovation diffusion and/ or adoption. Thus the gender and innovation diffusion and/ or adoption equation is not free from controversy. Thus future researchers still have to grapple with testing the research hypothesis to the effect that gender relates to the adoption of innovations in such a way that the males are more apt than the females.

\subsection{Income Level as a Correlate of Adoption of Innovations}

On the importance of income in innovation diffusion and/ or adoption, Schiffman and Kanuk (2004) observe that the "consumer innovators have...higher personal or family incomes and are more likely to have higher occupational statuses... than the late adopters or non-innovators" (p. 538). On his side, Rogers (2003) observes that the "earlier adopters... are wealthier.... [and] socio-economic status and innovativeness appear to go hand in hand" (p. 288). However Rogers (2003) poses a rhetorical question, namely that, "do innovators innovate because they are richer, or are they richer because they innovate?" (p. 288). While regretting that "this cause-and-effect question 
cannot be answered solely on the basis of available cross-sectional data" (p. 288), he contends that there are understandable reasons why social status and innovativeness vary together.

Some new ideas are costly to adopt and require large initial outlays of capital, with only the wealthy units in a social system being able to adopt these innovations. Recent studies on income as a positive correlate (or cost as a negative correlate) of the adoption of innovations (e.g. Dlodlo, 2009) are available. But so are those (e.g. Bakkabulindi, Mulumba, Aluonzi, Oketch \& Taibu, 2010) that do not support the hypothesis. Thus studies still have to empirically challenge the position by Rogers (2003) to the effect that the "earlier adopters have a higher social status than the late adopters, [where] status is indicated by such variables as income...." (p. 288). Hence the hypothesis: Income level is positively related to the adoption of innovations.

\section{Hypotheses on Perceived Innovation Characteristics as Correlates of Adoption of Innovations}

\subsection{Perceived Relative Advantage as a Correlate of Adoption of Innovations}

In his Innovation Diffusion Theory (IDT), Rogers (2003) defines the perceived relative advantage (PRA) of an innovation, as the degree to which the innovation is perceived as being better than the idea it supersedes, and is often expressed as economic profitability, and as conveying social prestige. PRA can also be measured in terms of the convenience and satisfaction (Kelleher \& Sweetser, 2012) that the innovation brings to the adopter or user. Eason (1988) refers to Rogers' PRA as "system functionality" (SF), which he (Eason) defines as the ability of a system or innovation "to perform in order that it can support the required range of organizational tasks" (p. 129). In his Technology Adoption Model (TAM), Davis (1989) refers to Rogers' PRA as the "perceived usefulness" (PU) of an innovation, which he (Davis) defines as the "prospective user's subjective probability that using a specific application system will increase his or her job performance" (cited in Gupta et al., 2008, p. 144).

More recent innovation adoption researchers (e.g. Venkatesh et al., 2003) in their Unified Theory of Acceptance and Use of Technology (UTAUT) have introduced yet a new term, namely "performance expectancy" (PE) to refer to Rogers' PRA and Davis' PU of an innovation. El-Gayar, Moran and Hawkes (2011) define PE as the degree to which a potential adopter believes that using an innovation will help him or her improve performance on the job. Nov and Ye (2009) refer to Rogers' PRA as "job relevance", which they define as the fit 
between an innovation and the job goals a user needs to accomplish. Thus clearly, apart from using different phrases, the terms perceived relative advantage (Rogers, 2003), system functionality (Eason, 1988), perceived usefulness (Davis, 1989), performance expectancy (Venkatesh et al., 2003) and job relevance (Nov \& Ye, 2009) are synonyms and are considered as such in this paper.

Rogers (2003) asserts that the greater the perceived relative advantage, system functionality, perceived usefulness or performance expectancy of an innovation, the more rapid its adoption. Recent past studies (e.g. Kelleher \& Sweetser, 2012) positively relating PRA and the use of innovations are very many. But studies giving a different result (e.g. Bakkabulindi, Osunsan, Kazibwe, Samanya \& Mabonga, 2010) can also be got. Such contentious empirical results make it right and fitting for future studies to put Rogers (2003)'s assertion to the effect that "the relative advantage of an innovation, as perceived by members of a social system, is positively related to its rate of adoption" (p. 233), to the test. Hence in this paper, it is accordingly being hypothesized that: perceived relative advantage (PRA) positively correlates with the adoption of innovations.

\subsection{Perceived Compatibility as a Correlate of Adoption of Innovations}

According to Rogers (2003), the perceived compatibility (PC) of an innovation is the degree to which the innovation is perceived as consistent with the existing values, past experiences, and needs of potential adopters. Rogers contends that an innovation can be compatible or incompatible with (i) the socio cultural values and beliefs (ii) the previously introduced ideas and/ or (iii) the client needs for the innovation. Perceived compatibility is positively related to an innovation's rate of use (Rogers, 2003) in that an innovation or new idea that is more compatible is less uncertain to the potential user and fits more closely with the individual's situation. Such compatibility helps the individual to give a meaning to the new idea so that it is regarded as more familiar. Recent theories such as the Unified Theory of Acceptance and Use of Technology, UTAUT (Venkatesh et al., 2003) use the term "facilitating conditions" (FC) to refer to Rogers' PC of an innovation.

They define FC as the "degree to which an individual believes that an organizational and technical infrastructure exists to support use of the system" (in Gupta et al., 2008, p. 146). Thus the terms perceived compatibility, PC (Rogers, 2003) and facilitating conditions, FC (Venkatesh et al., 2003) are considered as synonyms in this paper. Recent studies (e.g. Kelleher \& Sweetser, 2012), positively relating perceived compatibility (PC) and the use of innovations can be found. Ironically, such empirical support for the hypothesis aside, contrary findings (e.g. Bakkabulindi, Osunsan et al., 2010) can be cited. 
Hence empirically, support for and against the hypothesis is available. This suggests the need for further studies to test the postulation to the effect that: perceived compatibility (PC) positively correlates with the adoption of innovations.

\subsection{Perceived User Friendliness as a Correlate of Adoption of Innovations}

In his Innovation Diffusion Theory (IDT), Rogers (2003) preferred to use the opposite of "perceived user friendliness" (PUF), namely "perceived complexity" which he defined as the "degree to which an innovation is perceived as relatively difficult to understand and use" (p. 257). However to avoid using a term "perceived complexity" which has negative connotation, while the other three perceived characteristics (perceived relative advantage, compatibility and 'observability') have positive ones, in this paper, picking a cue from Eason (1988 p. 133), the opposite term, "perceived user friendliness" (PUF) has been used. PUF is the degree to which an innovation is perceived as relatively easy to understand and use. Eason also refers to Rogers' PUF as the "usability" of a system, which he defines as the system offering its functionality in such a way that the planned users will be able to master and exploit it without undue strain on their capacities and skills.

In his TAM, Davis (1989) refers to Rogers' PUF as the "perceived ease of use" (PEU) of an innovation, which he (Davis) defines as the "degree to which a prospective user expects the target system to be free of effort" (cited in Gupta et al., 2008, p. 144). More recent innovation adoption researchers (e.g. Venkatesh et al, 2003) in their Unified Theory of Acceptable and Use of Technology (UTAUT) have coined a new term "effort expectancy" (EE) to refer to Roger's PUF of an innovation. El-Gayar et al. (2011) define EE of an innovation as the degree of ease associated with the use of the innovation (i.e. the degree to which a potential adopter considers the use of the innovation to be free of effort).

Thus apart from using different phraseology, the terms perceived user friendliness (Eason, 1988; Rogers, 2003), perceived ease of use (Davis, 1989) and effort expectancy (Venkatesh et al, 2003) are the same and are used interchangeably in this paper. Al-Hajri and Tatnall (2008) contend that understanding perceived ease of use (PEU) is important because it has implication for the design of training intervention to manipulate the perception of PEU. Recent past studies that have positively related PUF and the use of innovations are many (e.g. Lee et al., 2009). However, other studies (e.g. Bakkabulindi, Osunsan et al., 2010) have found PUF not to correlate with innovation diffusion and/ or adoption. Hence in this paper it is being suggested that future researchers continue to test the hypothesis to the effect that 
perceived user friendliness (PUF) positively correlates with the adoption of innovations.

\subsection{Perceived 'observability' as a Correlate of Adoption of Innovations}

Perceived 'observability' (PO) is the degree to which results of an innovation are visible to others (Rogers, 2003). Thus PO can also be referred to as the perceived communicability or 'describability' of an innovation. Rogers observes that whereas some ideas are easily observed, communicated or described to other people, other innovations are difficult to observe, communicate or describe to others. For example, an innovation such as ICT, has two components; (i) hardware which are the physical parts of ICT, and (ii) software that consists of the instruction base for the technology. Thus the software component of a technological innovation (e.g. ICT) is not so apparent to observation. So innovations in which the software aspect is dominant possess less 'observability', and usually have a relatively slower rate of use (Rogers, 2003).

Awa et al. (2012) refer to Rogers' PO as the "perceived service quality" (PSQ) of an innovation, which according to them reflects an innovation's "image in customers' eyes, the overall customer judgment of the superiority or excellence of" the innovation or the "customer comparison between the actual and ideal performances of an application" (p. 577). According to them, PSQ can be measured in terms of cost effectiveness, customer satisfaction and customer retention among others. Awa et al. stress the importance of PSQ by asserting that its absence throws an adopter into psychological tensions, thus:

actual performance short of ideal performance throws the customer into psychological tensions...; feelings of tension and anxiety to balance cognitive elements and pains inflicted by anxiety. Avoiding exaggerated product claims or insisting on understanding product claims assists in making informed decisions leading to dissonance free exchanges (p. 577).

Nov and Ye (2009) refer to Rogers' PO as the "result demonstrability" (RD) of an innovation, which they defined as the tangibility of the results of using the innovation.

They assert that RD reflects the extent to which a user believes that the results of an innovation are discernible. Different phraseology notwithstanding therefore, the terms perceived 'observability' (Rogers, 2003), perceived service quality (Awa et al., 2012) and results demonstrability (Nov \& Ye, 2009) are the same and are used interchangeably in this paper. Several recent studies (e.g. Putzer \& Park, 2010) have positively related the perceived 'observability' (PO) and the use of innovations. However, most of these studies pertain to ICT innovations, thus suggesting that future studies especially those on other 
innovations than ICT, continue to test the hypothesis to the effect that perceived 'observability' (PO) positively correlates with the adoption of innovations.

\section{Hypotheses on Organisation Characteristics as Correlates of Adoption of Innovations}

\subsection{Organisational Readiness for Change as a Correlate of Adoption of Innovations}

Organisational readiness for change (RFC) is the "organisational members' beliefs, attitudes and intentions regarding the extent to which changes are needed and the organisation's capacity to successfully make those changes" (Bouckenooghe, 2010, p. 503). Organisational RFC therefore, can also be termed as organizational innovativeness or organisational ability to absorb change. Mullins (2010) observes that although organizations should have RFC, that is the readiness to adapt to their environments in order to survive, they tend to feel comfortable operating within the structure, policies and procedures which have been formulated to deal with the present situations. They thus set up defences or resistance to change (RTC) and prefer to concentrate on the routine things that they perform well. According to Avey, Wernsing and Luthans (2008), the resistance may take a number of forms such as dysfunctional attitudes (e.g. disengagement or cynicism) and behaviours (e.g. deviance).

Mullins (2010) attributes the RTC to organizational culture, the need to maintain stability, investment in the status quo, fear to disrupt past contracts or agreements and threats to power or influence the proposed change implies. On his part, Rogers (2003) attributes the RTC in organizations to bureaucracy where

rules are made and orders issued by individuals of authority and carried out by organizational members who accept the system of authority. At first, this control system operates in a rational and efficient manner, but the organizational effectiveness of bureaucracy is often lost over time. Rules are enforced overzealously and applied to all cases in an impersonal and inappropriate way. Bureaucratic leaders become impersonal, and the rationality of the system disappears. Nevertheless, organization members, trapped in an 'iron cage of control', continue to support the bureaucratic system (p. 405).

Several authors (e.g. Mooij \& Smeets, 2001) prescribe measures that managers can use to curb organizational RTC, including creating dissatisfaction in the 
organization with the status quo; by reducing the fear of change in the organization; by encouraging participation of all in the change effort; and by trying to compensate those affected by any change.

Studies positively relating organisational readiness for change (RFC) or negatively relating its opposite, resistance to change (RTC) and innovation adoption can be found (e.g. Aarons et al., 2009). Some studies (e.g. Nov \& Ye, 2008) however, have suggested that the RFC is only an indirect factor in the innovation adoption process, by showing that the resistance to change, RTC the opposite of RFC, was negatively related to the users' perceived ease of use, one of the key antecedents of technology adoption (Rogers, 2003). However, there are studies that discounted RFC as a correlate of innovation adoption (e.g. Bakkabulindi, 2012). Thus, because of the conflicting findings, future researchers are called upon to continue testing the hypothesis that organisational readiness for change (RFC) is positively correlated with the adoption of innovations.

\subsection{Organisational Culture as a Correlate of Adoption of Innovations}

Culture, a concept developed from Anthropology is difficult to define or explain precisely (Mullins, 2010). Nevertheless quite a few suggestions have come up. It has variously been conceptualized as; "how things are done around here"; as the "underlying assumptions about the way work is performed"; "what is acceptable and not acceptable"; "what behaviour and actions are encouraged and discouraged" (Mullins, 2010, p. 739). If change is to succeed in an organisation, one needs to understand the culture that is to be changed. If the proposed changes contradict the cultural biases and traditions, the changes will be difficult to embed in the organisation. Since cultures are difficult to change, organizational culture $(\mathrm{OC})$ is among the sources of the resistance to change (Rogers, 2003). Change management authorities (e.g. French \& Bell, 1990) discuss cultural values that facilitate change in organizations.

They include (i) a manager adopting a management style that allows for devolution of power from the top to the bottom; (ii) convincing employees that there are benefits in accepting change; (iii) achieving commitment to organizational goals through making employees participate in the change process; (iv) ensuring team work where a leader encourages increased participation, information sharing and collective decision making. He also advocates for (v) the valuing of each employee's contribution to change; (vi) the empowerment of employees to release their creativity, thereby promoting change; (vii) ensuring continuous learning, which will ensure organizational survival as it enhances ability to adapt to the environment. Studies relating OC to innovation adoption can be found (e.g. Bakkabulindi \& Sekabembe, 2010). Studies in support of the hypothesis aside, there are studies that totally 
dismissed the hypothesis to the effect that $\mathrm{OC}$ is a correlate of innovation adoption (e.g. Bakkabulindi, 2012). The conflicting results of the studies cited imply that the following hypothesis is still calling for the attention of future researchers: organisational ICT culture is positively correlated with adoption of innovations.

\subsection{Organisational Size as a Correlate of Adoption of Innovations}

According to Mullins (2010), the size of an organization can be defined and measured in different ways, although according to him, the most common indicator of organizational size is the number of persons employed by the organization. Rogers (2003) asserts that the "size of an organisation has consistently been found to be positively related to its innovativeness" (p. 409), that is the readiness for innovations or changes of its members, which he observes, "might seem surprising, given the conventional wisdom that smaller companies can be more flexible in their operations and freer of stifling bureaucracy" (p. 410). He goes on to rationalize why size is one of the best predictors of organisational innovativeness, thus

size is... a surrogate measure of several dimensions that lead to innovation: total resources, slack resources (defined as the degree to which an organisation has more resources than those required for its ongoing operations), employees' technical expertise, organisational structure, and so on.... These "lurking" variables may be a fundamental reason for the common finding that size and innovativeness are related (p. 411).

Awa et al. (2012) on their part, with support of past studies suggest that the adoption of innovations such as electronic commerce (EC) is slower amongst smaller institutions perhaps because of the relative lack of education about innovation potentials, lack of technological expertise and lack of economy of scale advantage and facilitating slacks, among other reasons. However, there is an opposing view, which considers large organizations as overly bureaucratic and hence more resistant to the use of innovations. For example, Jaidee and Beaumont (2003) with a bias towards the adoption of business to business (B2B) electronic commerce (EC) in small and medium enterprises (SMEs) observed that SMEs arguably have distinct advantages over the large organizations in adopting B2B and other types of EC. First, they are smaller, making them more nimble in decision making, and unlike large organizations encumbered with large bureaucracies, SMEs can make quicker decisions to engage in a particular market opportunity or to create a new product or service. Because of their size, they contend, SMEs are more dependent than other organizations on external sources of scientific and technological innovation. 
Consequently SMEs are better able to respond and more flexible in adjusting to market conditions and technology change than large organizations. In summary, "there is a continuing debate on the comparative advantages of large and small organizations; on whether 'bigger is best' or 'small is beautiful"" (Mullins, 2010, p. 589) with respect to the use of innovations. A few empirical studies (e.g. Hung et al., 2010) have established that "organizational size" has a significant influence on the adoption of innovations. Many more studies however, have totally dismissed organisation size as a correlate of innovation adoption (e.g. Bakkabulindi \& Oyebade, 2011). Despite lack of unanimity, for the sake of future researchers, this paper tentatively concurs with Rogers' (2003) assertion that "earlier adopters have larger-sized units (farms, schools, companies) than do later adopters" (p. 288), and postulates that organisational size positively correlates with the adoption of innovations.

\subsection{Organisational Leader's Change Management Style as a Correlate of Adoption of Innovations}

Leading change is one of the most important and difficult leadership responsibilities (Yukl, 2006). "The role of leadership at all levels of an organization... is paramount for spearheading innovation as a process and maintaining its momentum until innovation... occurs" (Crossan \& Apaydin, 2010, p, 1156). It is thus important for managers to understand the reasons for, and nature of, resistance and to adopt a clearly defined strategy for the initiation of change (Mullins, 2010). Change management can be subdivided into two approaches, namely planned change and emergency change approaches. Planned change is a deliberate pre-meditated move to alter the organisational status. It is change initiated and implemented by change leaders to either solve problems, to adapt to changes or to influence future changes. On the other hand unplanned or emergency change is not a sequential process. It is chaotic and often involves shifting of goals, discontinuation of activities and making of unexpected combinations of changes. For any change process to be successful however, it must be properly managed. For example Mullins (2010) stresses the need for a change manager to use a participatory change style if the change is to succeed, arguing that while in certain situations, it may be necessary to use hierarchical authority to impose change through an autocratic (Theory X) style of leadership, in most cases, change is more effective with a participative (Theory Y) style of leadership, where staff are kept fully informed of proposals, and are encouraged to adopt a positive attitude and have personal involvement in the implementation of change.

A host of other sources (e.g. Romme, 2010) recommend the use of Organisational Development (OD) as a model of managing planned change. In particular, Romme defines OD as "any practice that serves to deliberately 
improve problem solving and renewal processes in organisations" (p. 9). While recent studies (e.g. Hung, et al., 2010) have supported the hypothesis that good change leadership can stimulate innovation adoption, evidence to the contrary (e.g. Bakkabulindi \& Oyebade, 2011) can be found. Thus, findings on the "leader's change management style" as a correlate of innovation adoption are controversial. Nevertheless, future researchers may verify the hypothesis that organisational leader's change management style positively correlates with the adoption of innovations.

\section{$7 \quad$ Discussion}

This paper was intended to (i) give a full account of the Innovation Diffusion Theory (IDT) as a framework for guiding studies on the correlates of innovation diffusion and/ or adoption; (ii) critique the more recent technology adoption models, namely the Technology Acceptance Model (TAM), the Technology-Organisation-Environment (TOE) framework and the Unified Theory of Acceptance and Use of Technology (UTAUT), and to argue that all of them are derivatives of the IDT and hence (iii) to make a call for a return to the IDT as the original theory for guiding research on innovation diffusion and/ or adoption by suggesting a framework based on the IDT, and to derive 14 hypotheses for future research, basing on a review of recent empirical literature. It has achieved those objectives. In particular the framework suggested, divides the correlates of innovation diffusion and/ or adoption into three categories, namely the individual adopter characteristics, the perceived innovation characteristics, and the social system or organizational characteristics.

In terms of the individual adopter characteristics, it has been hypothesized that the interaction with change agents, of relevance, training, and cosmopolitanism positively relate to the adoption of innovations by an individual, while age negatively relates to the adoption of innovations by the individual. Gender has been postulated to relate to the adoption of innovations in such a way that the males are more apt adopter than the females. The income level of an individual has been hypothesized to be positively related to the adoption of innovations by the individual. Regarding perceived innovation characteristics, the perceived relative advantage, compatibility, user friendliness and 'observability' of an innovation, have been postulated to positively relate to the adoption of the innovation in question.

On the social system or organizational characteristics, it has been postulated that each of organizational readiness for change, culture, size and leader's change management style positively relates to the adoption of innovations by individuals in the organisation. The review that led to the 14 hypotheses 
established several gaps in past studies that future researches can close. For example few studies on the diffusion and/ or adoption of innovations have been done outside the developed world (see, for example, the review by Awa et al., 2012). Further, the review led to the conclusion that some theories such as the Technology Adoption Model (TAM) of Davis (1989), are on the verge of being over-researched, while some such as the Unified Theory of Acceptance and Use of Technology (UTAUT) of Venkatesh et al. (2003) are hardly used.

Hence the need to diversify by picking more potential correlates of innovation diffusion and/ or adoption from their "reservoir", namely Rogers' Innovation Diffusion Theory (IDT) by avoiding or going beyond the two constructs, namely perceived usefulness (PU) and perceived ease of use (PEU) of TAM as advised by several authorities (e.g. Awa et al., 2012). Thus in addition to the perceived innovation characteristics, organisational characteristics and individual adopter characteristics will also be considered. The diversification being called for, will be in line with Rogers (2003) who contends that "the challenge for future research is to expand... and search for different objectives than those of the past. Perhaps there is need to dig deeper in directions that theory suggests" (p. 101). Otherwise, desirable though it is, the inclusion of all the possible correlates of innovation diffusion and/ or adoption in a given study is an impossibility, and hence a shortcoming of all studies.

That is why most studies acknowledge it in a language typical of the one used by Sim, Tan, Ooi and Lee (2011), thus; "like all studies... regarding... technology adoption, it is not possible to include all adoption factors in the model... Given that, it might be helpful if future studies consider the inclusion of additional variables in their investigation...." (p. 10). Further, most studies have been quantitative, a tradition introduced by rural sociologists (Rogers, 2003 , p. 53), and hence based on self-reporting questionnaires (Sang et al., 2010, p. 109). The data resulting from such self-reporting were based entirely on the honesty of the answers from the participants yet the participants may have provided less-than-accurate responses (Norton, 2012), although the same source observes that the use of anonymous questionnaires in many studies may have reduced the likelihood of this bias.

Pituch and Lee (2006) assert that the use of self-reporting measures "raises the possibility of common method variance, which may inflate the true associations between variables" (p. 239). Such shortcomings of self-reporting measures such as questionnaires, prompted Sang et al. (2010) to call for the use of more direct qualitative measures in future studies, thus, "future studies could build on... observation... and/ or interviews.... (p. 109). Rogers (2003, pp. 4850) observes that this qualitative approach to diffusion studies was the tradition of anthropologists, but has been generally neglected for some time now. Yet, as advised by Kelleher and Sweetser (2012), a "qualitative approach seems most appropriate to allow more in-depth discussion of the factors influencing not 
only adoption but also active use of [innovations such as] social media, which require human participation" (p. 109). Other advocates of the qualitative approach to studies on innovation diffusion and/ or adoption include Aarons et al. (2009).

Most studies have been cross-sectional or snapshot in nature, which restricted the full understanding of the decision making process with regard to the diffusion and/ or adoption of innovations. Rogers (2003) critiques such studies by observing that "cause-and-effect question[s] cannot be answered solely on the basis of... cross-sectional data" (p. 288). It is thus recommended that future researchers replicate earlier cross-sectional studies longitudinally, to identify the dynamics among factors critical to the said decision making (Hung et al., 2010, p. 601). In other words, time should be considered as an important variable in the innovation diffusion and/ or adoption process (Kelleher \& Sweetser, 2012). Another major challenge cutting across almost all studies was the issue of inadequate sample size and/ or sampling methods (e.g. see Hung et al., 2010).

Such inadequate sample sizes and/ or sampling methods restricted the generalization of the findings. Future studies thus have the challenge of refining the sampling methods to be used in innovation diffusion and/ or adoption studies. Many studies acknowledged the limitation of inadequate instruments. For example after their study of the influence of system characteristics on elearning use, Pituch and Lee (2006) critiqued their instruments by observing that, "better measures of system and user attributes should be developed, as we had to delete several items from these scales to attain good psychometric properties" (p. 239). Future studies thus have the challenge of refining the instruments used in innovation diffusion and/ or adoption studies (MacKenzie, Podsakoff \& Podsakoff, 2011). Perhaps, we should expect more papers in the genre of Sato and Zouain (2012) geared toward instrument development and/ or refinement.

\section{Conclusion}

The paper called for a return to Rogers' Innovation Diffusion Theory (IDT) as the original theory for guiding research on innovation diffusion and/ or adoption by suggesting a framework based on the IDT, and derived 14 hypotheses for future research, basing on a review of recent empirical literature. The framework suggested, divides the correlates of innovation diffusion and/ or adoption of into three categories, namely the individual adopter characteristics, the perceived innovation characteristics, and the social system or organizational characteristics. The model suggested however, has limitations. For example, it 
suggests hypotheses relating to only six individual adopter characteristics, namely the interaction with change agents, training, cosmopolitanism, age, gender and income level, yet other individual characteristics such as selfefficacy, professional experience and workload (Buabeng-Andoh, 2012) of the potential adopter, exist.

Only four perceived innovation characteristics, namely the perceived relative advantage, computability, user friendliness and 'observability' were considered in the model, excluding others such as the perceived trialability and risk (Rogers, 2003) of an innovation. Only four social system or organizational characteristics, namely the organizational readiness for change, culture, size and leader's change management style were used in the framework, excluding several other social system or organizational characteristics such as the availability of training of relevance to the innovation, accessibility to, and technical support (Awa et al., 2012) with respect to the innovation. Future researchers can thus expand the model. Nevertheless, hopefully the paper has contributed to solidifying the theoretical/ conceptual foundation on which future papers, both theoretical and empirical will build.

\section{References}

Aarons, G. A., Sommerfield, D. H., \& Walrath-Greene, C. M. (2009). Evidence-based practice implementation: Impact of public versus private sector organisation type on organisational support, provider attitudes and adoption of evidence-based practice. Implementation Science, 4 (1). doi: 10.1186/ 1748-5908-4-83.

Al-Hajri, S., \& Tatnall, A. (2008). Technological innovation and adoption of internet banking in Oman. Electronic Journal for Virtual Organisations and Networks, 10, $59-83$.

Avey, J. B., Wernsing, T. S., \& Luthans, F. (2008). Can positive employees help positive organizational change? Impact of psychological capital and emotions on relevant attitudes and behaviours. Journal of Applied Behavioural Science, 44 (1), 48-70. doi: 10.1177/ 0021886307311470.

Awa, H. O., Ukoha, O., \& Emecheta, B. C. (2012). Integrating TAM and TOE frameworks and expanding their characteristic constructs for e-commerce adoption by SMEs. Proceedings of Informing Science \& IT Education Conference (InSITE), pp. 571-588.

Bakkabulindi, F. E. K. (2011). Individual characteristics as correlates of use of ICT in Makerere University. International Journal of Computing and ICT Research, 5 (2), 38 - 45. Retrieved from: http:// www.ijcir.org/ volume5number2/ article4.pdf. 
Bakkabulindi, F. E. K. (2012). How do organisational characteristics relate with use of knowledge management systems? Makerere Journal of Higher Education, 4 (1), 71-81.

Bakkabulindi, F. E. K., \& Kabasiita, J. (2012). Change agents, training and age as correlates of use of computers among Masters students in Makerere University School of Education. Nkumba Business Journal, 12, 145-163.

Bakkabulindi, F. E. K., Mulumba, F. N., Aluonzi, B., Oketch, C., \& Taibu, A. (2010). Readiness for open and distance education of doctoral students in Kampala International University. Journal of Science and Sustainable Development, 3 (1), 51-56.

Bakkabulindi, F. E. K., Osunsan, K. O., Kazibwe, S., Samanya, B., \& Mabonga, E. (2010). Use of computers by doctoral students in Kampala International University as a function of perception. Journal of Educational Review, 3 (4), 461-467. Retrieved from: http:// www.serialspublications.com/ contentnormal.asp? jid $=472 \&$ jtype $=1$.

Bakkabulindi, F. E. K., \& Oyebade, S. (2011). Organisational characteristics and use of personal computer software by graduate students in Makerere University. Journal of US-China Education Review, A3, 331 - 338.

Bakkabulindi, F. E. K., \& Sekabembe, B. (2010). Age, gender and organisational culture as correlates of use of knowledge management systems in Makerere University. In A. Tatnal, O. C. Kereteletswe, \& A. Visscher (Eds.), Information technology and managing quality education (pp.30-42). Boston, US: Springer. Retrieved from: http://www.springerlink.com/ content/ k26g078024jv2502.

Billon, M., Macro, R., \& LeraLopez, F. (2009). Disparities in ICT adoption: Multidimensional approach to study cross-country digital divide. Telecommunications Policy, 33, 596-610. doi: 10.1016/ j.telpol.2009.08.006.

Bouckenooghe, D. (2010). Positioning change recipients' attitudes toward change in organizational change literature. Journal of Applied Behavioural Science, 46 (4), 500-531. doi: 10.1177/ 0021886310367944.

Buabeng-Andoh, C. (2012). Factors influencing teachers' adoption and integration if ICT with teaching: Review of literature. International Journal of Educational Development using ICT, 8 (1), 136 - 155.

Crossan, M. M., \& Apaydin, M. (2010). Multi-dimensional framework of organizational innovation: Systematic review of literature. Journal of Management Studies, 47 (6), 1154-1191. doi: 10.1111/ j.14676486.2009.00880.x.

Davis, F. D. (1986). Technology Acceptance Model for empirically testing new end-user information system: Theory and results. Doctoral dissertation, Sloan School of Management, Massachusetts Institute of Technology, Massachusetts, US. 
Davis, F. D. (1989). Perceived usefulness, perceived ease of use, and user acceptance of information technology. MIS Quarterly, 13 (3), 319 - 340.

Dlodlo, N. (2009). Access to ICT education for girls and women in rural South Africa: A case study. Technology in Society, 31, 168 - 175. doi: 10.1016/ j.techsoc.2009.03.003.

Eason, K. (1988). Informational technology and organisational change. London, UK: Taylor and Francis.

Educause Centre for Applied Research, ECAR. (2010). Technology adoption and ownership of IT. In ECAR. (Eds.), Students and Information Technology (pp. 37 - 53).

El-Gayar, O., Moran, M., \& Hawkes, M. (2011). Students' acceptable of tablet PCs and implications for educational institutions. Educational Technology \& Society, 14 (2), $58-70$.

French, W. L., \& Bell, C. H. Jr. (1990). Organizational development: Behavioural science interventions for organization improvement $\left(4^{\text {th }}\right.$ ed.). New Jersey, US: Prentice-Hall International.

Gupta, B., Dasgupta, S., \& Gupta, A. (2008). Adoption of ICT in a government organisation in a developing country: Empirical study. Strategic Information Systems, 17, 140 - 154.

Hong, E. N. C., Hao, L. Z., Kumar, R., Ramendran, C., \& Kadiresan, V. (2012). Effectiveness of human resource management practices on employee retention in an institute of higher learning: Regression analysis. International Journal of Business Research and Management, 3 (2), 60-79.

Hung, S., Hung, W., Tsai, C., \& Jiang, S. (2010). Critical factors of hospital adoption on CRM system: Organisational and information system perspectives. Decision Support Systems, 48, 592 - 603. doi: 10.1016/ j.dss.2009.11.009.

Jaidee, S., \& Beaumont, N. (2003). Factors affecting SME owners/managers in adoption of business-to-business techniques: Research framework. Monash, Australia: Monash University Business and Economics, Department of Management Working Series. Working Paper 47/03. Retrieved from: www.monash.edu.au/ mgt/ research/ working-papers/ 2003/ wp47-03.pdf.

Kelleher, T., \& Sweetser, K. (2012). Social media adoption among university commentators. Journal of Public Relations Research, 24 (2), 105 - 122. doi: 10.1080/ 1062726x.2012.626130.

Korpelainen, E. (2011). Theories of ICT system implementation: A critical review. Helsinki, Finland: Department of Industrial Engineering and Management, School of Science and Technology, Aalto University. Retrieved from: www.aalto.fi.

Lee, B., Yoon, J., \& Lee, I. (2009). Learners' acceptance of e-learning in South Korea: Theories and results. Computers \& Education, 53, 1320 - 1329. doi: 10.1016/ j.compedu.2009.06.014. 
Lule, I., Omwansa, T. K., \& Waema, T. M. (2012). Application of the Technology Acceptance Model (TAM) to m-banking adoption in Kenya. International Journal of Computing and ICT Research, 6 (1), 31 - 43.

MacKenzie, S. B., Podsakoff, P. M., \& Podsakoff, N. P. (2011). Construct measurement and validation procedures in MIS [management information systems] and behavioural research: Integrating new and existing techniques. MIS Quarterly, 35 (2), 293 - 344.

Mooij, T., \& Smeets, E. (2001). Modelling and supporting ICT implementation in secondary schools. Computers \& Education, 36, 265 - 281.

Mullins, L. J. (2010). Management and organizational behaviour. London, UK: Pitman.

Norton, E. (2012). Exploratory study to examine intentions to adopt evidencebased HIV linkage-to-care intervention among state health department AIDS directors in the United States. Implementation Science, 7. Retrieved from: http:// www.implementationscience.com/ content/ 7/ 1/ 27.

Nov, O., \& Ye, C. (2008). Users' personality and perceived ease of use of digital libraries: The case of resistance to change. Journal of American Society for Information Science and Technology, 59 (5), 845 - 851.

Nov, O., \& Ye, C. (2009). Resistance to change and adoption of digital libraries: An integrative model. Journal of American Society for Information Science and Technology, 60 (8), $1702-1708$.

Oliveira, T., \& Martins, M. F. (2011). Literature review of information technology adoption models at firm level. Electronic Journal of Information Systems Evaluation, 14 (1), 110 - 121. Retrieved from: www.ejise.com.

Pituch, K. A., \& Lee, Y. (2006). Influence of system characteristics on elearning use. Computers \& Education, 47, 222 - 244.

Putzer, G. J., \& Park, Z. (2010). Effects of innovation factors on smartphone adoption by nurses in community hospitals. Perspectives in a Health Information Management, Winter. Retrieved from: http:// www.perspectives.ahima.org/index.php?option.

Rogers, E. M. (1957). Conceptual variable analysis of technological change. Doctoral dissertation, Ames, Iowa State University, Ames, Iowa, US.

Rogers, E. M. (2003). Diffusion of innovations ( $5^{\text {th }}$ ed.). New York, US: Free Press.

Rogers, E. M., Singhal, A., \& Quinlan, M. M. (2009). Diffusion of innovations. In D.W. Stacks, \& M. Salwen (Eds.), An integrated approach to communication theory and research ( $\left.2^{\text {nd }} \mathrm{ed}, \mathrm{pp} .418-434\right)$. Mahwah, New Jersey, US: Lawrence Erlbaum Associates.

Romme, A. G. L. (2010). Organisational development interventions: Artifaction perspective. Journal of Applied Behavioural Science, 47(8), 8-32. doi: $10.1177 / 0021886310390864$. 
Salleh, F., Yaakub, N., \& Dzulkifli, Z. (2011). Influence of skill levels on job performance of public service employees in Malaysia. Business and Management Review, 1 (1), 31-40.

Sang, G., Valcke, M., van Braak, J., \& Tondeur, J. (2010). Student teachers' thinking processes and ICT integration: Predictors of prospective teaching behaviours with educational technology. Computers \& Education, 54, 103 112. doi: 10.1016/ j.compedu.2009.07.010

Sato, R. C., \& Zouain, D. M. (2012). Factor analysis for adoption of nuclear technology in diagnosis and treatment of chronic diseases. Einstein, 10 (1), $62-63$.

Schiffman, L. G., \& Kanuk, L. L. (2004). Consumer behaviour $\left(8^{\text {th }}\right.$ ed.). New Delhi, India: Prentice - Hall of India.

Ssali, S. N., Ahikire, J., \& Madanda, A. (2007). Gender concepts handbook (popular version). Kampala, Uganda: Gender Mainstreaming Division, Makerere University.

Sim, J. J., Tan, G. W. H., Ooi, K. B., \& Lee, V. H. (2011). Exploring individual characteristics on adoption of broadband: An empirical analysis. International Journal of Network and Mobile Technologies, 2 (1). Retrieved from: http:/ijnmt.intimal.educ.my.

Stuart, L. H., Mills, A. M., \& Remus, U. (2009). School leaders, ICT competence and championing innovations. Computers \& Education, 53, 733 - 741. doi: 10.1016/ j.compedu.2009.04.013.

Tornatzky, L. G., \& Fleischer, M. (1990). Processes of technological innovation. Lexington, Massachusetts, US: Lexington Books.

Venkatesh, V., Morris, M. G., Davis, G. B., \& Davis, F. D. (2003). User acceptance of information technology: Toward a unified view. MIS Quarterly, 27 (3), 425 - 478.

Williams, M. D., Rana, N. P., Dwivedi, Y. K., \& Lal, B. (2011). Is UTAUT really used or just cited for the sake of it? Systematic review of citations of UTAUT's originating article. Proceedings of ECIS (Paper 231). Retrieved from: aislel.aisnet.org/ ecis2011/ 231.

Yukl, G. (2006). Leadership in organizations ( $6^{\text {th }}$ Ed.). New Jersey, US: Prentice-Hall. 